\title{
Adiponectin and Type 1 Diabetes Mellitus in Children
}

\author{
PSN MENON \\ Consultant \& Head, Department of Pediatrics, Jaber-Al-Ahmed Armed Forces Hospital, PO Box 5819, Salmiya, Kuwait. \\ psnmenon@yahoo.com
}

$\mathrm{T}$ here is an explosion of information related to adiponectin in the last decade. We know that adipose tissue secretes many adipocytokines of which adiponectin modulates a number of vital metabolic processes related to glucose homeostasis and fatty acid catabolism [1]. It is exclusively secreted from adipose tissue into the blood and is abundant in plasma at levels of 5-10 $\mu \mathrm{g} / \mathrm{mL}$. Girls have higher levels than boys. Levels of the hormone are inversely correlated with BMI and body fat percentage in adults. The association in infants and young children is less clear.

Genomic studies have helped us to understand the action of adiponectin better. Adiponectin self-associates into larger structures. Three adiponectin oligomers bind together to form a trimer; trimers self-associate to form hexamers, dodecamers or high molecular mass (HMW) isoforms consisting of at least 12 to 18 protomers. Thus adiponectin circulates in at least three different subforms. Each isoform of adiponectin exerts distinct biological properties in target tissues [2]. Recent studies indicate that HMW oligomer may be the most biologically active form concerning glucose homeostasis, whereas the central actions are attributed to the low molecular weight oligomers [3].

Adiponectin modulates a number of metabolic processes including those resulting in type 2 diabetes (T2DM), obesity, coronary artery disease and metabolic syndrome. Contrary to expectations, adiponectin is decreased in obesity. The association of low adiponectin levels with obesity and hyperinsulinemia has been confirmed in cross sectional studies in 5 and 10 year old children; however, the association with hyperinsulinemia is not completely independent of obesity [4]. Probably adiponectin plays a less important role in whole body insulin sensitivity in children.

In adults with T2DM, circulating levels of HMW adiponectin are selectively decreased due to an impaired secretion of this oligomer from adipocytes [5]. Their levels increase after weight reduction and following bariatric surgery. Single-nucleotide polymorphisms in the adiponectin gene associated with low plasma adiponectin levels and T2DM have been identified.

The story is different in type 1 diabetes (T1DM). Adult T1DM patients, especially with diabetic nephropathy have elevated total levels of the adiponectin unlike T2DM. Adiponectin probably increases as a compensatory response in these patients with microvascular complications [4]. Renal failure may lead to the stimulation of adiponectin production as a physiological response to restrict endothelial damage. It may also decrease adiponectin clearance, and the kidney may develop secondary resistance to adiponectin. Still high total adiponectin levels were predictive of development of microalbuminuria in T1DM in some studies suggesting a causal association [1]. Studies on large cohorts of healthy subjects also have shown that adiponectin levels increase before the onset of nephropathy.

Recent studies have shown that the levels of adiponectin remain higher in persons with type 1 diabetes than in non-diabetics, even after controlling for renal function, obesity and HDL cholesterol. The absolute concentrations of total adiponectin and all subforms were higher in T1DM patients than healthy controls. This increase in concentration of total adiponectin was primarily caused by a major increase of the HMW sub-form. This association was not associated with gender or diabetic nephropathy status [2].

Compared to adults, studies in children are limited. A longitudinal study from Germany showed that children and adolescents with T1DM have BMI-dependent elevated serum adiponectin compared with healthy children [6]. There were similar observations in obese diabetic boys in an earlier study published in Indian Pediatrics [7]. In this issue of the journal, Habeeb, et al. provide another observation of adiponectin as a marker for complications in children with T1DM [8].

How these deleterious effects of adiponectin are induced is not well understood. In vitro experiments have suggested that adiponectin may increase NFkB 
production. HMW oligomer may be protective with inhibition of $\mathrm{NFkB}$, whereas the low and medium subforms are associated with nephropathy. Altered glycosylation of lysine leading to changed adiponectin function has been postulated as another mechanism.

Has adiponectin come of age as a routine test or as a predictor of obesity, T2DM, T1DM or even development of microangiopathy or comorbidities? Not yet. There are simpler and better clinical methods now. To some extent it may have role in adult T2DM. Its utility in T1DM, especially children, is far from clear.

Funding: None; Competing interests: None stated.

\section{REFERENCES}

1. Díez JJ, Iglesias P. The role of the novel adipocyte-derived hormone adiponectin in human disease. Eur J Endocrinol. 2003;148:293-300.

2. Leth H, Andersen KK, Frystyk J, Tarnow L, Rossing P, Paeving $\mathrm{HH}$, et al. Elevated levels of high-molecularweight adiponectin in type 1 diabetes. J Clin Endocrinol Metab. 2008;93:3186-91.
3. Oh DK, Ciaraldi T, Henry RR. Adiponectin in health and disease. Diabetes Obes Metab. 2007; 9:282-9.

4. Stefan N, Bunt JC, Salbe AD, Funahashi T, Matsuzawa Y, Tataranni PA. Plasma adiponectin concentrations in children: relationships with obesity and insulinemia. J Clin Endocrinol Metab. 2002; 87: 4652-6.

5. Weyer C, Funahashi T, Tanaka S, Hotta K, Matsuzawa Y, Pratley RE, et al. Hypoadiponectinemia in obesity and type 2 diabetes: close association with insulin resistance and hyperinsulinemia. J Clin Endocrinol Metab. 2001; 86:1930-5.

6. Galler A, Gelbrich G, Kratzsch J, Noack N, Kapellen T, Kiess W. Elevated serum levels of adiponectin in children, adolescents and young adults with type 1 diabetes and the impact of age, gender, body mass index and metabolic control: a longitudinal study. Eur J Endocrinol. 2007;157:481-9.

7. El-Mesallamy HO, Hamdy NM, Ibrahim SM. Adiponectin and pro-inflammatory cytokines in obese diabetic boys. Indian Pediatr. 2011; 48: 815-6.

8. Habeeb NMM, Youssef OI, Saab AA, El Hadidi ES. Adiponectin as a marker of complications in children with type 1 diabetes. Indian Pediatr. 2012;49:277-80.

\title{
Thalassemia: Cardiac Iron and Chelators
}

\author{
AJAy Gaur \\ Department of Pediatrics, GR Medical College, Gwalior, 474 001, Madhya Pradesh, India. \\ drajaygaur@gmail.com
}

I ron overload in thalassemia is a serious and potentially fatal condition as excess iron is toxic to tissues and organs, particularly the liver and the heart. The serum ferritin level is useful in assessing iron balance trends, but does not accurately predict quantitative iron stores. Measurement of the iron level by liver biopsy is the standard method for accurately determining the iron store. A ferritometer and specialized MRI software are emerging alternatives for liver biopsies. Although quantitative liver iron measurement accurately guides the use of iron chelators, it may not reflect cumulative changes in cardiac iron. Thalassemics may have cardiac iron overload even at the time of a safe liver iron measurement [1].

Cardiac damage caused by iron overload is the main cause of death in thalassemia. An increased risk of ironinduced cardiac disease is observed with liver iron concentration (LIC) values above $15 \mathrm{mg}$ of iron per gram of dry weight of liver, and in patients with serum ferritin values above 2500 microgram/liter. The rate of iron loading depends mainly on the rate of blood transfusions, which causes a net iron deposition in the body, of about 15-20 mg/day. In practice, the goal of chelation therapy is to achieve an iron balance by accessing two iron pools, namely intracellular labile iron pool (LIP) and iron from red cell catabolism [2].

After the introduction of deferoxamine in 1963, several efforts were made to synthesize orally active iron chelators. Following the screening of more than 700 chelators from various chemical classes, deferasirox emerged as a highly selective chelator for iron with high oral potency and tolerability. Deferasirox mobilizes iron stores by binding selectively to the ferric form of iron and enters most of the cells to reach the major intracellular sites of iron accumulation. For myocardial iron, deferasirox has the ability to enter myocardial cells and chelate iron from these cells. It was also observed from myocyte cultures that it rapidly gains entry in the myocytes and binds to labile intracellular iron, leading to decreased free radical production.

In recent years, clinical trials have been conducted to evaluate the effect of deferasirox on myocardial iron and 
the left ventricular ejection fraction (LVEF). Of the 23 patients who received deferasirox $10-30 \mathrm{mg} / \mathrm{kg} /$ day, for $13.1( \pm 0.78)$ months; the mean myocardial $\mathrm{T} 2 *$ measurement was inversely related to myocardial iron content. Deferasirox treatment led to significant reductions in mean serum ferritin concentrations and LICs, while no changes in LVEF were noted [3]. Study conducted by Pathare, et al. [4] monitored cardiac siderosis using T2 MRI in 19 heavily iron overloaded patients with $\beta$-thalassemia major receiving iron chelation therapy with deferasirox over an 18 months period. Deferasirox therapy significantly improved means cardiac T2 from a baseline of 17.2 (10.8) to 21.5 (12.8) ms. A concomitant reduction in median serum ferritin, and mean LIC was also noted. Improvements were seen in patients with various degrees of cardiac siderosis, including myocardial iron in those patients with a baseline cardiac T2 of $<10 \mathrm{~ms}$, indicative of high cardiac iron burden [4].

Studies on long term use, dose, efficacy and safety profile of deferasirox have concluded that deferasirox in doses of $20-30 \mathrm{mg} / \mathrm{kg} /$ day could effectively reduce iron burden [5]. Further, efficacy of available chelators on myocardial iron and biventricular function by quantitative MRI in 550 thalassemics concluded that oral deferasirox has better global systolic ventricular function compared to oral deferiprone and subcutaneous desferoxamine [6].

In the background of such global studies, this prospective, open label, single arm study on 30 patients by Merchant, et al. [7] reported good safety profile of deferasirox, and showed that it effectively chelates myocardial iron, more efficacious in moderate to severe cardiac iron overload. In addition, there was a significant decrease in serum ferritin in those patients with cardiac $\mathrm{T} 2 *<10 \mathrm{~ms}$ and between $10-20 \mathrm{~ms}$. Similar cardiac findings have also been reported by other researchers
[4]. Although the sample size is small, this study adds that for Indian population, deferasirox is a safe and efficacious iron chelator without any significant adverse effect even with doses of $>30 \mathrm{mg} / \mathrm{kg} /$ day. Thus, the data shows promising results of deferasirox on cardiac iron and quantifies myocardial iron by non-invasive method. Presently, many thalassemia centers monitor cardiac iron with T2 weighted MRI imaging, but routine application of this technology has not been implemented across all centers.

Funding: None; Competing interests: None stated.

\section{REFERENCES}

1. Michael RD, Jones MF, Vichinsky E. Thalassemia syndrome. In: Kliegman RM, Stanton B, Schor NF, St. Geme III JW, Behrman RE, editors. Nelson Textbook of Pediatrics. $19^{\text {th }}$ ed. Philadelphia: Elsevier, Saunders; 2011. p.1675-76.

2. Agarwal MB. Deferasirox: Oral, once daily iron chelatorAn expert opinion. Indian J Pediatr. 2010;77:185-191

3. Neufeld EJ. Oral chelators deferasirox and deferiprone for transfusional iron overload in thalassemia major: new data, new questions. Blood. 2006;107:3436-41.

4. Pathare A, Taher A, Daar S. Deferasirox (Exjade ${ }^{\circledR}$ ) significantly improves cardiac T2* in heavily iron overload patients with â thalassemia major. Ann Hematol. 2010,89:405-9.

5. Nisbet Brown E, Olivieri NG, Giardina PJ, Grady RW, Neufeld EJ, Sechaud R, et al. effectiveness and safety of placebo controlled, dose escalation trial. Lancet. 2003;361:1597-1602.

6. Pepe A, Meloni A, Capra M, Clanciulli P, Prossomariti L, Malaventura $\mathrm{C}$, et al. Deferasirox, deferiprone and desferoxamine treatment in thalasemia major patients: cardiac iron and function comparison determined by quantitative magnetic resonance imaging. Haematologica. 2011;96:41-7.

7. Merchant R, Ahmed J, Krishnan P, Jankharia B. Efficacy and Safety of deferasirox for reducing total body and cardiac iron in thalasemia. Indian Pediatr. 2012;49:281-5.

\title{
Public Health Significance of Shigellosis
}

\author{
Sujit K Bhattacharya, *Dipika Sur and Dilip Mahalanabis \\ Society for Applied Studies, Salt Lake; and *National Institute of Cholera and Enteric Diseases, Kolkata, India. \\ sujitkbhattacharya@yahoo.com
}

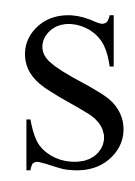

higellosis is an important intestinal infection of public health concern, accounting for 140 million cases globally per year and 60000 deaths annually of which $60 \%$ occur in children below 5 years of age [1]. The disease can occur as sporadic, epidemic and pandemic forms. The disease has a short incubation period. In 1969-1970, an epidemic of shigellosis caused by multi-drug resistant $S$.dysenteriae type 1 occurred in Central America and rapidly spread to different parts of Africa and Asia. The epidemic was seen 
in Bangladesh in 1970s and in Eastern India in 1974 [2]. The disease is characterized by fever, loose stools mixed with blood and mucus, tenesmus and abdominal cramps. Dehydration is not generally a conspicuous feature. Shigellosis is caused by four species of Shigella viz., S.sonnei, S.flexneri, S. boydii and S.dysenteriae. S.sonnei causes mild dysentery in developed countries, while $S$. dysenteriae type 1 causes severe dysentery in developing countries in patients with poor hygiene, sanitation and improper disposal of human and animal waste and overcrowding. Shigellosis can occur in high risk populations, viz., displaced populations, travellers, in the military and day-care centers. Each of them are subdivided into several serotypes, e.g., S.flexneri 1-6, S. boydii 1-18, S.sonnei phase I and phase II, and S.dysenteriae 1-12. Three strains are responsible for causing majority of shigellosis cases, viz., S. sonnei, S. flexneri $2 a$ and $S$. dysenteriae Type 1.

In the article on school outbreak of S. sonnei infection in China in this issue of the journal [3], S. sonnei strains exhibited high degree of drug resistance. Usually, shigellosis caused by $S$. dysenteriae type 1 is characterized by multiple drug resistance and high morbidity and mortality particularly in children below 5 years of age [4]. $S$. dysenteriae type 1 may be associated with a number of complications like rectal prolapse, leukemoid reaction, convulsions and hemolytic uremic syndrome (HUS). In this study, the shigella strains were sensitive to ciprofloxacin and third generation cephalosporins. In view of the reported cartilage toxicity of fluroquinolones in animal model, the drug was not used in China where it is prohibited for use in children. However, in many countries, fluroquinolones are used in children successfully for the treatment of infections, without any cartilage toxicity being reported $[3,6]$. High rate of antimicrobial resistance as well as high prevalence of class 2 integrons among $S$. sonnei species was observed in this study. The authors suggest that it is mandatory to continuously monitor the local antibiotic resistance patterns of Shigella species [7]. However, it is imperative to keep in mind that stool cultures are often negative, more so, if the sample has been processed long after collection.

Hand washing with plenty of water with soap or mud, improvement of environmental sanitation, water supply and avoidance of overcrowding are required for prevention of the disease, particularly in slums and refugee camps. Vaccine development for shigellosis is a formidable task as it can be caused by a number of serotypes and immunity to Shigella is serotype specific. Several attempts have been made to develop a safe and effective vaccine against shigellosis, but none is yet available for public use.

Competing interests: None; Funding: Nil.

\section{REFERENCES}

1. Kotloff KL, Winckloff JP, Ivanoff B, Clemens JD, Swerdlow DL, Sansonetti PJ, et al. Global burden of Shigella infections: implications for vaccine development and implementation of control strategies. Bull World Health Org. 1999;77:651-6.

2. Rahaman MM, Khan MM, Aziz KM, Islam MS, Kibriya AK. An outbreak of dysenteriae type 1 on a coral island in Bay of Bengal. J infect Dis. 1975;132:15-9.

3. Xiao GG, Fan J, Deng JJ, Chen CH, Zhou W, Li XH, et al. A school outbreak of Shigella sonnei infection in China: clinical features, antibiotic susceptibility and molecular epidemiology. Indian Pediatr. 2012; 49:287-90.

4. Bhattacharya SK, Sur D. An evaluation of current shigellosis treatment. Expert Opin Pharmacotherapy. 2003;4:1315-20.

5. Bhattacharya SK, Sarkar K, Nair GB, Faruque AS, Sack DA. Multidrug-resistant Shigella dysenteriae type1 in south Asia. Lancet Infect Dis. 2003;3:755.

6. Khan WA, Seas C, Dhar U, Salam MA, Bennish ML. Treatment of Shigellosis: V. Comparison of azithromycin and ciprofloxacin-A double-blind, randomized, controlled trial. Ann Intern Med. 1997;126:697-703.

7. Sur D, Niyogi SK, Sur S, Datta KK, Takeda Y, Nair GB, et al. Multidrug-resistant Shigella dysenteriae type 1: forerunners of a new epidemic strain in eastern India? Emerg Infect Dis. 2003;9:404-5. 